\title{
Characterization of the Intrinsic Fibrinolytic Properties of Pro-urokinase through a Study of Plasmin-resistant Mutant Forms Produced by Site-specific Mutagenesis of Lysine ${ }^{158}$
}

\author{
Victor Gurewich, Ralph Pannell, Robert J. Broeze,“ and Jen-i Mao* \\ Vascular Laboratory, Departments of Vascular Medicine and Biomedical Research, St. Elizabeth's Hospital; Tufts University School of \\ Medicine, Boston, Massachusetts 02135; and *Collaborative Research, Inc., Bedford, Massachusetts 01730
}

\begin{abstract}
Two plasmin-resistant mutant forms of pro-urokinase (proUK) constructed by site-directed mutagenesis of Lys $^{158}$ to $\mathrm{Val}^{158}$ and $\mathrm{Met}^{158}$ were used to evaluate the intrinsic enzymatic and fibrinolytic properties of pro-UK as distinct from those of its two-chain UK (TC-UK) derivative. Both mutants, while resistant to plasmin activation, were as sensitive as pro-UK to degradation by thrombin. Since thrombin cleaves a peptide bond only two residues from the activation site, the integrity of this loop was maintained in the two mutants. The amidolytic and plasminogen-activating activities of the mutants averaged 0.14 and $0.12 \%$ that of TC-UK, respectively. The fibrin plate activities were $2,400 \mathrm{IU} / \mathrm{ml}$ and $700 \mathrm{IU} / \mathrm{mg}$ for the $\mathrm{Met}^{158}$ and $\mathrm{Val}^{158}$ mutants or about $1.5 \%$ that of TC-UK. These findings attest to a discrete but low intrinsic activity for pro-UK and suggest that the higher values reported in the literature may be related to UK contaminants or plasmin-induced TC-UK generation during the assay. Clot lysis by the mutants required doses $>100$-fold higher than those of pro-UK to induce a comparable effect. From this it appears that pro-UK activation is a major determinant of the rate of clot lysis occurring with proUK. Clot lysis by the mutants was potentiated by plasmin pretreatment of the fibrin and by the addition of small amounts of TC-UK or tissue plasminogen activator (t-PA). Combinations of t-PA and the mutants were synergistic in their fibrinolytic effects. These findings mirror those previously obtained with pro-UK. We concluded that the previously described potentiation of pro-UK-induced clot lysis by UK or t-PA is mediated primarily by pro-UK itself rather than by a promotion of its activation.
\end{abstract}

\section{Introduction}

Pro-urokinase (pro-UK) ${ }^{1}$ is a single-chain, 55,000 $M_{\mathrm{r}}$ plasminogen activator that is a precursor of two-chain, high molecular weight urokinase (TC-UK). Like TC-UK (1) pro-UK was

Address reprint requests to Dr. Victor Gurewich, Vascular Laboratory, St. Elizabeth's Hospital, 736 Cambridge Street, Boston, MA 02135. 1988

Received for publication 20 May 1988 and in revised form 19 July

1. Abbreviations used in this paper: DFP, diisopropylfluorophosphate; n-pro-UK, native pro-urokinase; pro-UK, pro-urokinase; TC-UK, two-chain urokinase; t-PA, tissue plasminogen activator; UK, urokinase.

J. Clin. Invest

(C) The American Society for Clinical Investigation, Inc. 0021-9738/88/12/1956/07 \$2.00

Volume 82, December 1988, 1956-1962 originally isolated from urine, but almost 30 years later (2-4). It has subsequently been purified from human cell culture media and from plasma (5-8). Specific cleavage of the Lys ${ }^{158}$ Ile $^{159}$ peptide bond of pro-UK (9) results in its activation to TC-UK. Both plasmin $(8,10)$ and kallikrein $(11,12)$ will activate pro-UK.

Since plasmin is the product of plasminogen activation and an activator of pro-UK, it greatly complicates measurement of the intrinsic activity of pro-UK against plasminogen. Several different methodologies have been used in an effort to isolate the primary reaction of pro-UK with plasminogen, and a wide range of activities has been reported (13-15). As a result, the relative contributions of pro-UK and TC-UK to fibrinolysis remain unclear. When pro-UK conversion to TC-UK was monitored with a radiolabeled probe during clot lysis in a plasma milieu only $\sim 10 \%$ conversion was observed (16). This experiment was inconclusive but suggested that the small proportion of TC-UK generated may have been incidental to fibrinolysis that was induced by pro-UK itself.

To study the enzymatic and fibrinolytic properties of proUK in isolation from those of TC-UK, plasmin-resistant mutants of recombinant pro-UK were constructed by site-specific mutagenesis of $\mathrm{Lys}^{158}$ to $\mathrm{Val}^{158}$ or to $\mathrm{Met}^{158}$. The mutants were expressed in a mammalian cell system.

\section{Methods}

\section{Cloning the human $c D N A$ gene for pro- $U K$}

Restriction enzymes, T4 DNA ligase, T4 polynucleotide kinase, calf alkaline phosphatase, and Escherichia coli DNA polymerase I (Klenow fragment) were obtained from New England Biolabs (Beverly, MA) or Boehringer Mannheim Biochemicals (Indianapolis, IN).

Total cytoplasmic RNA was isolated from $5 \mathrm{~g}$ of frozen TCL-598 cells (17) using the procedure of Favaloro et al. $(18,19)$. cDNA libraries, prepared in a single-stranded phage fl vector according to the method described by Moir et al. (20), were transferred to nitrocellulose filters and screened by plaque hybridization (21) using synthetic oligonucleotides (15- or 20-mers) based on the published nucleotide sequence of a human pro-UK cDNA (22). A full-length copy of TCL598 pro-UK cDNA gene was constructed by ligating the restriction fragments from two overlapping clones. The coding region for pro-UK was sequenced by the dideoxy (23) and the Maxam-Gilbert (24) methods.

Construction of Lys ${ }^{158}$ derivatives of pro-UK

The full-length pro-UK cDNA was inserted into the mammalian expression vector pSV2 (25) to yield plasmid pCGM16. The construction of a cDNA encoding the pro-UK-Val ${ }^{158}$ mutant (carried by plasmid pCGM28) is illustrated in Fig. 1. Briefly, the DNA coding sequence for amino acid residues 153-164 (a small Dde I/Eco RI fragment) in pCGM 16 was replaced by two synthetic oligonucleotides (a 30- and a 31-mer). In the synthetic sequence the codon specifying Lys ${ }^{158}$ was replaced by one specifying valine and a unique Sac II restriction site was introduced. Plasmid pCGM30, which carried mutant cDNA-en- 


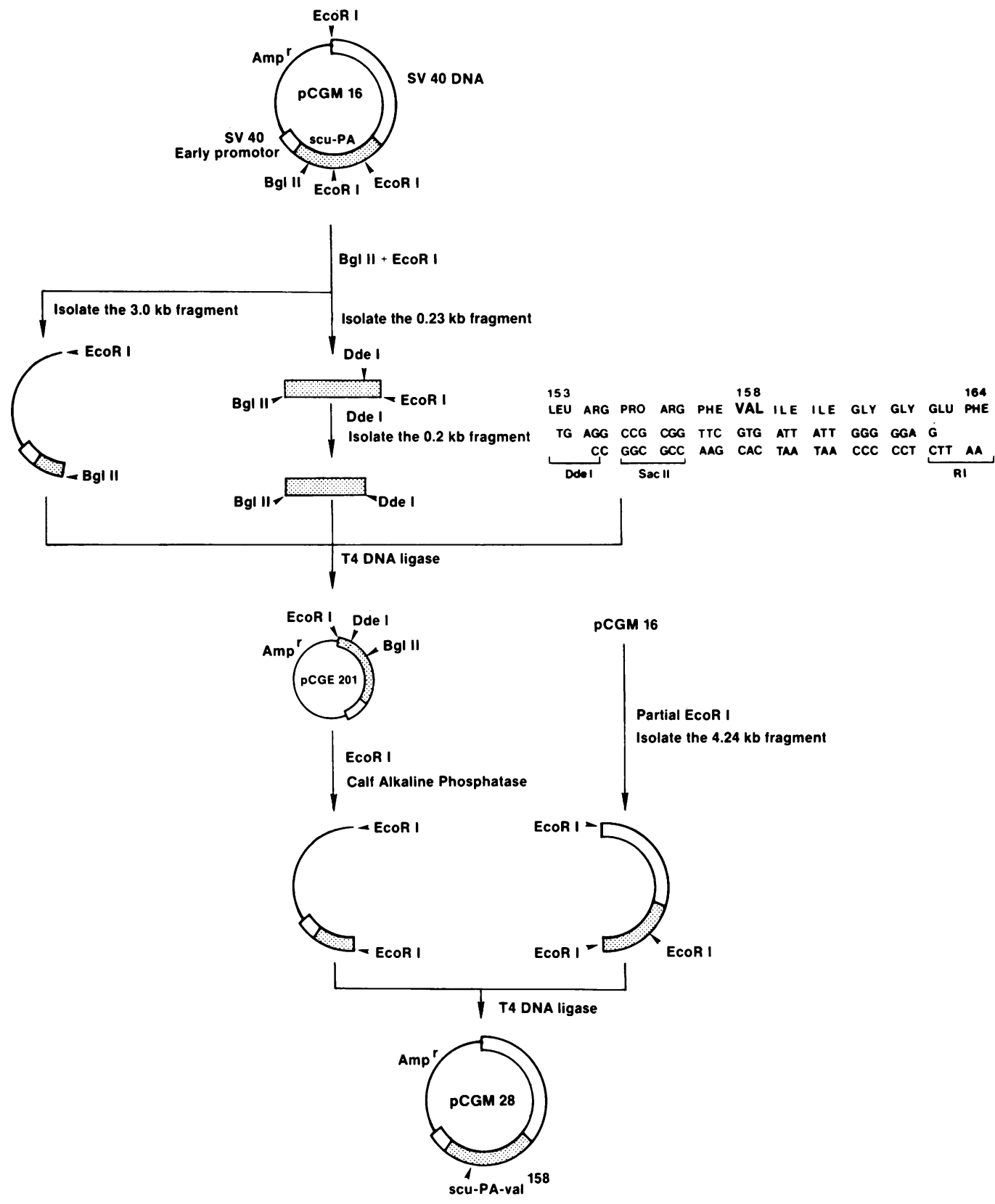

Figure 1. Construction of the pro-UK-(scu-PA)$\mathrm{Val}^{158}$ expression plasmid. The resulting plasmid pCGM28 contains pBR322 sequences (line), the SV40 early promoter (open bar), a pro-UK (scu-PA) cDNA derivative encoding pro-UK(scu-PA)-Val ${ }^{158}$ (stippled bar), and additional sequences from the SV40 early region (open bars).

coding pro-UK-Met ${ }^{158}$, was derived by replacing the small Sac II/ Eco RI fragment of pCGM27 with synthetic oligonucleotides. The nucleotide sequences of these pro-UK derivatives were confirmed by dideoxy or Maxam-Gilbert sequencing.

\section{Transfection and gene amplification}

The mammalian host cell line used for expression of the pro-UK derivatives was Chinese hamster ovary cell strain DG44, which lacks the diploid DHFR locus (26). The expression vectors for the Lys ${ }^{158}$ mutants were cotransfected with pSV2-DHFR (27) or plasmid pdhfr2.9 (28) by the calcium coprecipitation procedure (29). Transfectants were selected in Ham's F-12 medium (Gibco Laboratories, Grand Island, NY) supplemented with $10 \%$ dialyzed fetal bovine serum (Gibco Laboratories), but lacking glycine, hypoxanthine, and thymidine. They were individually expanded and screened for production of protein antigenically related to pro-UK/TC-UK by an ELISA.

To increase expression levels, transfectants producing pro-UK derivatives were subjected to an amplification procedure that involved challenging cells with increasing concentrations of MTX (30). This procedure was terminated when a yield of about 2-10 mg/liter of each pro-UK derivative was obtained. Amplified cells were grown to confluency in roller bottles and fed with DME (Gibco Laboratories) and Ham's F-12 medium mixed at a ratio of $1: 1$ and supplemented with MTX and 2\% fetal bovine serum. Conditioned medium was harvested every 3-4 d.

\section{ELISA for pro-UK/TC-UK antigen present in conditioned medium}

An ELISA that used a polyclonal antibody raised in rabbits against urinary TC-UK was used to quantitate pro-UK/TC-UK-related antigen present in conditioned medium produced by Chinese hamster ovary cells. $100 \mu \mathrm{l}$ conditioned medium was incubated in wells of a microtiter plate for $3 \mathrm{~h}$ at $37^{\circ} \mathrm{C}$. UK antigen bound to the wells was quantitated using an anti-TC-UK-plasminogen activator-horseradish peroxide conjugate and the substrate ABTS. A standard curve was constructed by plotting the concentration of urinary UK standards 
(diluted in nonconditioned medium) vs. absorbance at $405 \mathrm{~nm}$. This was used to quantitate the amount of pro-UK or pro-UK derivative present in the samples.

\section{Purification of pro-UK derivatives}

Conditioned medium was filtered through a $0.2-\mu \mathrm{m}$ pleated capsule filter (Gelman Sciences, Inc., Ann Arbor, MI), titrated to pH 6.0 with hydrochloric acid, and applied to a column of S Sepharose Fast Flow (Pharmacia Fine Chemicals, Piscataway, NJ) equilibrated with $20 \mathrm{mM}$ sodium phosphate buffer, $\mathrm{pH} 6.0$, and $0.15 \mathrm{M}$ sodium chloride. After washing with equilibration buffer the column was eluted with $20 \mathrm{mM}$ sodium phosphate and $0.5 \mathrm{M}$ sodium chloride, $\mathrm{pH}$ 6.0. Fractions were pooled based on their absorbance at $280 \mathrm{~nm}$ and the presence of pro-UK-related antigen as quantitated by ELISA.

Eluates from S Sepharose were adjusted to $1.0 \mathrm{M}$ sodium chloride, pH 7.0, and then applied to pABZ-Sepharose (Collaborative Research, Inc., Lexington, MA) equilibrated with $20 \mathrm{mM}$ sodium phosphate and $1 \mathrm{M}$ sodium chloride, $\mathrm{pH}$ 7.0. The column was washed with $20 \mathrm{mM}$ sodium phosphate and $1 \mathrm{M}$ sodium chloride, $\mathrm{pH} 7.5$, and then eluted with $0.1 \mathrm{M}$ sodium acetate and $0.1 \mathrm{M}$ sodium chloride, $\mathrm{pH}$ 4.8. Protein that eluted at $\mathrm{pH} 4.8$ was pooled based on absorbance at $280 \mathrm{~nm}$ and the presence of UK-related antigen as determined by ELISA.

Small amounts of contaminating two-chain material derived from the cleavage of pro-UK derivatives after $\mathrm{Arg}^{156}$, present in the pABZ eluates, were removed by immunoaffinity chromatography using an anti-pro-UK-specific monoclonal antibody resin. The pABZ-Sepharose eluate was titrated to $\mathrm{pH} 7.2$ and applied to the immunoaffinity resin. After washing the column with $0.02 \mathrm{M}$ sodium phosphate, $\mathrm{pH}$ 7.2 , and $0.15 \mathrm{M}$ sodium chloride, the single-chain pro-UK derivative was eluted from the column with $50 \mathrm{mM}$ glycine, $\mathrm{pH}$ 2.0. The flowthrough fractions containing the contaminating two-chain material were discarded. Protein was quantitated using the method of Lowry et al. (31).

\section{Other proteins and reagents}

Native pro-UK (n-pro-UK) purified from the culture medium of a transformed human kidney cell was obtained from Collaborative Research, Inc. Two-chain UK was a product of Green Cross (Osaka, Japan). The World Health Organization International Reference preparation of UK, 66/46 (IRP-UK), was used as the activity standard. Tissue plasminogen activator (t-PA), predominantly single chain, was obtained from Integrated Genetics (Framingham, MA). Fibrinogen and the plasmin substrate (S2251) were obtained from Kabi AB (Stockholm, Sweden). BSA was purchased from Calbiochem-Behring Corp. (San Diego, CA). The UK substrate ( $p$ Glu-Gly-Arg-p-nitroanaline) was obtained from American Diagnostica (New York, NY).

Plasminogen-free fibrinogen was prepared from Kabi fibrinogen by diisopropylfluorophosphate (DFP; $5 \mathrm{mM}$ ) treatment followed by chromatography on lysine-Sepharose equilibrated to $5 \mathrm{mM}$ trisodium citrate and $0.15 \mathrm{M} \mathrm{NaCl}$. The product was then dialyzed against $0.05 \mathrm{M}$ Hepes, pH 7.4 and $0.15 \mathrm{M} \mathrm{NaCl}$.

Pooled plasma ( $3 \mathrm{U})$ was obtained from St. Elizabeth's Hospital Blood Bank (Boston, MA). Plasminogen was purified from DFPtreated human plasma essentially by the method of Castellino and Powell (32).

UK was repurified on benzamidine-Sepharose and immobilized on Actigel-A (Sterogene, San Gabriel, CA). Plasmin was prepared from purified plasminogen by activation with immobilized UK in HBSS/50\% glycerol. Purified human thrombin was a gift from Dr. John Fenton (Albany, NY).

\section{Physical characterization of the Lys ${ }^{158}$ mutants}

Sensitivity to plasmin and thrombin. A reaction mixture of $2 \mu \mathrm{M}$ $\mathrm{n}$-pro-UK or mutant pro-UK and plasmin $(0.01 \mu \mathrm{M}$ for n-pro-UK and $0.1 \mu \mathrm{M}$ for the $\mathrm{Met}^{158}$ and Val ${ }^{158}$ mutants) in HBSS-Tween was incubated at $37^{\circ} \mathrm{C}$ for $60 \mathrm{~min}$. Thrombin $(0.1 \mu \mathrm{M})$ treatment for $60 \mathrm{~min}$ at $37^{\circ} \mathrm{C}$ was also carried out under the same conditions. The results were examined by $12 \%$ SDS-PAGE under reducing conditions (15).
Enzymatic activity of the mutants. The amidolytic activities of the mutants were measured with synthetic substrate as previously described (15) and compared with those of similar quantities of TC-UK and n-pro-UK.

The plasminogen-activating activities were measured in a reaction mixture containing the plasmin substrate (S2251), $0.5 \mu \mathrm{M}$ Glu-plasminogen, $1 \mathrm{mg} / \mathrm{ml} \mathrm{BSA,} 0.05 \mathrm{M}$ Hepes, $\mathrm{pH} 7.4$, and $0.1 \mathrm{M} \mathrm{NaCl}$. The reaction mixture was equilibrated to $37^{\circ} \mathrm{C}$ before addition of the activator $(50 \mathrm{ng} / \mathrm{ml} \mathrm{UK}, 1$ and $2.5 \mu \mathrm{g} / \mathrm{ml} \mathrm{n}$-pro-UK). Absorbance (405 $\mathrm{nm}$ ) was monitored by chart recorder. The second derivative of the curve of absorbance was used to calculate the relative rate of plasminogen activation. The activities of the mutants were compared with those of TC-UK.

The specific activity of the mutants was measured on a fibrin plate against the UK reference standard 66/46 (National Institute of Biological Standards and Control, London).

\section{Clot lysis}

${ }^{125} \mathrm{I}$-labeled fibrin clots were prepared from $0.25 \mathrm{ml}$ plasminogen-free fibrinogen $(5 \mathrm{mg} / \mathrm{ml})$. After the addition of $1.5 \mu \mathrm{Ci}{ }^{125}$ I-fibrinogen (fibrin, human fibrinogen; Amersham Corp., Arlington Heights, IL) clotting was induced with $1 \mathrm{NIH}$ unit/ml thrombin plus $5 \mathrm{mM} \mathrm{CaCl}$. The clots were formed in 5-mm (Id) glass tubing, incubated for $30 \mathrm{~min}$ at $37^{\circ} \mathrm{C}$, and refrigerated overnight as previously described (10). The next morning the clot was transferred by careful reaming of the glass tube with a 21-gauge spinal tap needle to $1 \mathrm{ml}$ plasma. After incubation at $37^{\circ} \mathrm{C}$ for $30 \mathrm{~min}$, the plasma was removed with a Pasteur pipette and the clot transferred to the incubation mixture containing the constituents to be tested in a total vol of $1 \mathrm{ml}$ plasma. Lysis was quantitated from the release of radioactivity and expressed as a percent of the $100 \%$ clot lysis value.

For some experiments fibrin clots were pretreated with plasmin as previously described (35). In brief, $1 \mathrm{ml}$ of plasmin solution $(2 \mu \mathrm{g} / \mathrm{ml}$ in $0.05 \mathrm{M}$ Hepes, pH 7.4, $0.15 \mathrm{M} \mathrm{NaCl}, 1 \mathrm{mg} / \mathrm{ml} \mathrm{BSA}$ ) for $30 \mathrm{~min}$ at $25^{\circ} \mathrm{C}$. Degree of digestion was estimated from the release of radioactivity. The reaction was stopped by incubation at $37^{\circ} \mathrm{C}$ in plasma for 30 min which arrested release of radioactivity.

At the end of each experiment fibrinogen was determined as thrombin-clottable protein and plasminogen was assayed using synthetic substrate (S-2251) as previously described (16).

\section{Results}

\section{Physical characterization of the Lys ${ }^{158}$ mutants}

Both Lys ${ }^{158}$ substitution mutants comigrated with n-pro-UK on reducing SDS-PAGE (Fig. $2 A$, lanes 1-3). However, whereas plasmin induced conversion of n-pro-UK to TC-UK as previously described (16), the mutants resisted cleavage by plasmin even at 10 times the concentration required to cleave n-pro-UK (Fig. 2 B, lanes 2, 3). By contrast, the mutants retained the sensitivity of n-pro-UK to cleavage by thrombin (Fig. $2 C$, lanes 1-3). Thrombin induces degradation of n-proUK to an inactive TC-UK (33) by cleaving the $\mathrm{Arg}^{156}-\mathrm{Phe}^{157}$ bond, two residues from the activation site (12).

\section{Characterization of the activities of the Lys ${ }^{158}$ mutants}

Compared with TC-UK, the Met ${ }^{158}$ and Val ${ }^{158}$ mutants had activities of 0.08 and $0.17 \%$, respectively. This activity is similar to the $0.4 \%$ previously reported for n-pro-UK when it was measured in the presence of inhibitors to UK and plasmin (15) (Table I).

The specific activities measured on fibrin plates were 2,400 and $700 \mathrm{IU} / \mathrm{mg}$ for the $\mathrm{Met}^{158}$ and $\mathrm{Val}^{158}$ mutants, respectively, or 2.4 and $0.7 \%$ that of TC-UK based on a TC-UK activity of $100,000 \mathrm{IU} / \mathrm{mg}$ (Table I). These activities are simi- 


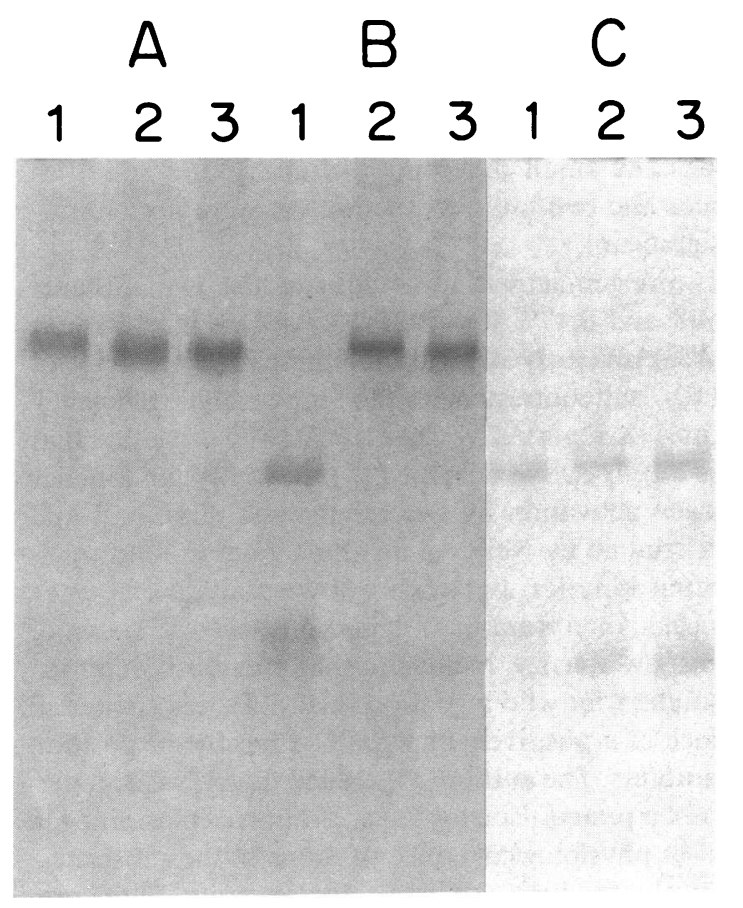

Figure 2. SDS-PAGE (12\% gels) under reducing condition. 1, n-proUK $(2 \mu \mathrm{M}) ; 2, \mathrm{Met}^{158}(2 \mu \mathrm{M})$ mutant; 3 , Val ${ }^{158}(2 \mu \mathrm{M})$ mutant. $(A)$ control; $(B)$ after plasmin $(0.01 \mu \mathrm{M}$ for n-pro-UK and $0.1 \mu \mathrm{M}$ for the mutants) treatment for $1 \mathrm{~h}\left(37^{\circ} \mathrm{C}\right) ;(C)$ after thrombin $(0.1 \mu \mathrm{M})$ treatment for $60 \mathrm{~min}\left(37^{\circ} \mathrm{C}\right)$. For the latter, $1 \mu \mathrm{M}$ of the pro-UK preparations was used.

lar to those recently reported for Gly ${ }^{158}$ and Glu ${ }^{158}$ pro-UK mutants that were 800 and $530 \mathrm{IU} / \mathrm{mg}$, respectively (34).

\section{Clot lysis studies}

Clot lysis by 10,20 , and $30 \mu \mathrm{g} / \mathrm{ml}$ of either mutant induced modest but dose-responsive lysis that went to completion at 5 $\mathrm{d}$ only at the highest dose. The clot lysis curves obtained with the Met ${ }^{158}$ mutant are shown (Fig. $3 \mathrm{~A}$ ). A comparable lytic effect was induced by only $0.01-0.03 \mu \mathrm{g} / \mathrm{ml}$ of n-pro-UK. Moreover, in contrast to lysis with the two mutants, clot lysis went to completion at all doses of n-pro-UK (Fig. $3 \mathrm{~B}$ ). Therefore, under these conditions the nonactivatable Lys ${ }^{158}$ mutant forms of pro-UK were $\sim 0.3-1 \%$ as active as the n-pro-UK.

Effect of plasmin pretreatment of clots on lysis by mutant pro-UK. Limited plasmin pretreatment of fibrin clots has previously been shown to attenuate the lag phase and greatly potentiate clot lysis by n-pro-UK (35). Pretreatment of clots with plasmin sufficient to induce $\sim 1 \%$ lysis based on release of radioactivity consistently potentiated clot lysis by both mu-

Table I. Enzymatic Activities Expressed as a Percent of TC-UK Activity

\begin{tabular}{lll}
\hline & \multicolumn{2}{c}{ Lys $^{158}$ mutants } \\
\cline { 2 - 3 } & $\mathrm{Met}^{158}$ & $\mathrm{Val}^{158}$ \\
\hline Amidolytic & 0.08 & 0.2 \\
Plasminogen activator & 0.08 & 0.17 \\
Fibrin plate & 2.4 & 0.7 \\
\hline
\end{tabular}

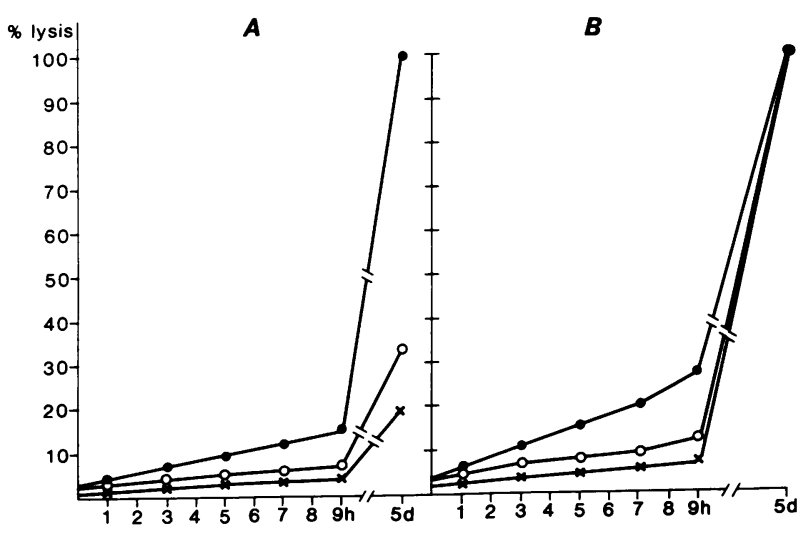

Figure 3. Lysis of ${ }^{125}$ I-fibrin clots in plasma by $(A) 10(\times), 20(0)$, or $30 \mu \mathrm{g} / \mathrm{ml}(\bullet)$ of the Met ${ }^{158}$ mutant pro-UK and by $(B) 0.01(\times), 0.02$ (O), or $0.03 \mu \mathrm{g}$ of n-pro-UK.

tants. The mean and range of the radioactivity released during clot lysis from five experiments with $30 \mu \mathrm{g} / \mathrm{ml}$ of the $\mathrm{Val}^{158}$ mutant are shown (Fig. 4).

Effect of UK on clot lysis by the mutants. Small amounts of UK have also previously been shown to potentiate clot lysis by n-pro-UK (36). When $10 \mathrm{IU} / \mathrm{ml}$ of TC-UK was added at 0 and $1 \mathrm{~h}$ to a plasma incubation mixture containing a clot and 30 $\mu \mathrm{g} / \mathrm{ml}$ of either mutant, the rate of lysis was significantly potentiated. After $9 \mathrm{~h}$ clot lysis was $<20 \%$ without and $>50 \%$ with UK. The UK alone induced little ( 15\%) clot lysis (Fig. 5). Control tubes containing no activator invariably showed $<10 \%$ apparent lysis and averaged $5 \%$ lysis at $9 \mathrm{~h}$ based on release of radioactivity (data not shown).

Effect of $t-P A$ on clot lysis by the mutants. The combination of t-PA with n-pro-UK under certain experimental conditions has been shown to be synergistic in fibrinolysis in vitro (35-37), though this interpretation has been disputed (38). When t-PA was combined with $30 \mu \mathrm{g} / \mathrm{ml}$ of either mutant a dramatic potentiation of clot lysis was seen. In the experiment illustrated, $30 \mu \mathrm{g} / \mathrm{ml}$ of the $\mathrm{Val}^{158}$ mutant in combination with 20 or $30 \mathrm{ng} / \mathrm{ml} \mathrm{t}$-PA induced 88 and $100 \%$ clot lysis in $9 \mathrm{~h}$, respectively. This compared with 30 and $38 \%$, respectively, for 20 and $30 \mathrm{ng} / \mathrm{ml}$ of $\mathrm{t}-\mathrm{PA}$ alone and a mean of $13 \%$ (range 9.5-15\%) from five experiments for the mutant alone (Fig. 6, $A, B)$.

These combinations of mutant pro-UK and t-PA appeared to be more than additive in their fibrinolytic effect. However, according to the strict definition of synergy proposed by Berenbaum (39) equivalent effects by fractional combinations of $<1$ compared with either agent alone must be shown. Accord-

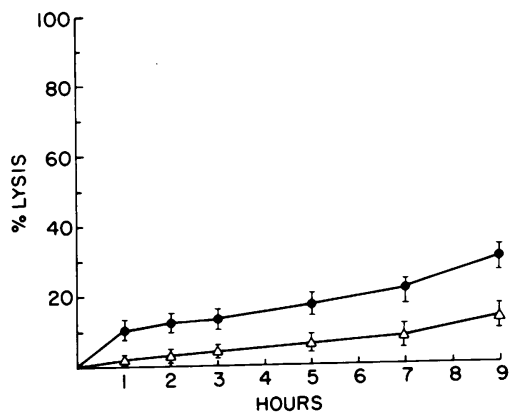

Figure 4. Lysis of ${ }^{125} \mathrm{I}-\mathrm{fi}-$ brin clots from five experiments (mean and range shown) by the $\mathrm{Val}^{158}$ mutant ( 30 $\mu \mathrm{g} / \mathrm{ml})$. Control clots $(\Delta)$ and fibrin clots pretreated by limited exposure to plasmin (๑). 


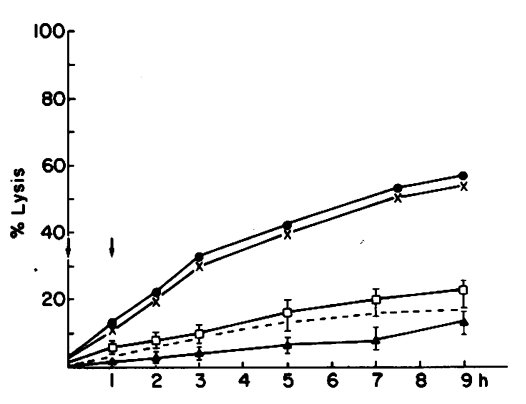

ingly, $30 \mathrm{ng} / \mathrm{ml} \mathrm{t}$-PA plus $30 \mu \mathrm{g}$ of the mutant induced lysis comparable to that by $120 \mathrm{ng}$ of t-PA alone. Unfortunately, insufficient amounts of either mutant were available to determine how much was required to induce $100 \%$ clot lysis with the mutant alone. However, a comparable lytic effect could be obtained with $600 \mathrm{ng} / \mathrm{ml}$ of n-pro-UK (data not shown). Since the mutant was found to be $0.3-1 \%$ as active (see above) it may be estimated that $60-180 \mu \mathrm{g} / \mathrm{ml}$ of mutant would be required. Accordingly, the sum of the fractional amount of mutant $(0.16-0.5)$ and of t-PA $(0.25)$ to achieve a comparable lytic effect of either one alone would be $0.41-0.75$. Since this sum is $<1$ it fulfills the definition of synergy.

At the end of each of the clot lysis experiments the plasminogen and fibrinogen concentrations in the plasma were measured and found to be not significantly changed from their baseline values.

\section{Discussion}

Two nonactivatable mutant forms of pro-UK were constructed by site-directed mutagenesis of $\operatorname{Lys}^{158}$ to study the enzymatic and fibrinolytic properties of pro-UK in functional isolation. The mutants were insensitive to plasmin even at 10 times the concentration that activates n-pro-UK, but were degradable by thrombin, like n-pro-UK (33). Since thrombin cleaves the molecule only two residues away from the activa-

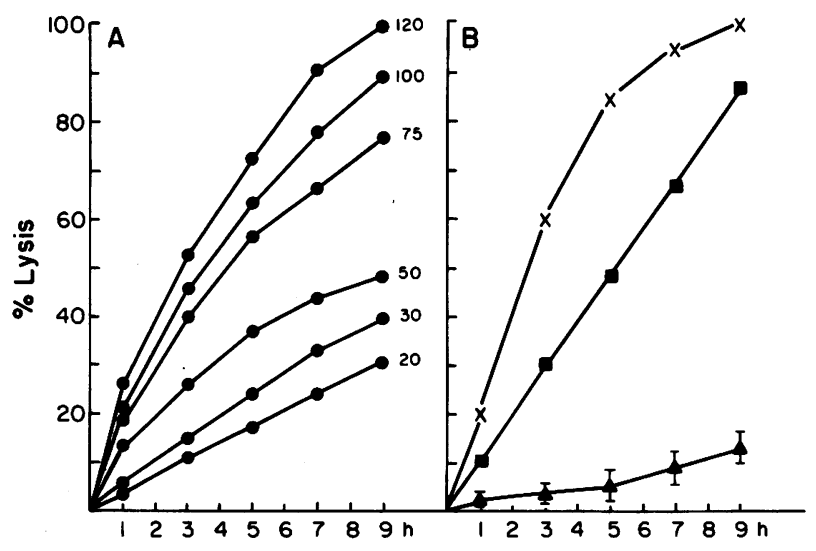

Figure 6. Lysis of $\mathrm{I}^{-125}$ fibrin clots in plasma. $(A)$ Lytic effect of a dose range of $20-120 \mathrm{ng} / \mathrm{ml}$ of single-chain t-PA. (B) Lysis by Val ${ }^{158}$ mutant pro-UK $(30 \mu \mathrm{g} / \mathrm{ml})$ alone ( $\triangle$ ) or in combination with 20 $\mathrm{ng} / \mathrm{ml}(\boldsymbol{\square})$ or $30 \mathrm{ng} / \mathrm{ml}(x)$ of t-PA. tion site (12), the findings suggest that the exposure of this loop is maintained in the mutants. The apparent integrity of the mutants was also seen in their amidolytic activities which were found to be comparable to that previously reported for $n$-proUK $(10,16)$. The small differences in enzymatic activities found between the two mutants themselves were not considered to be significant.

The plasminogen-activating activity of the two mutants was only 0.08 and $0.17 \%$ that of TC-UK. This is consistent with the $0.4 \%$ previously reported for the relative activity of n-pro-UK (10), but contrasts with the high catalytic efficiency $\left(\mathrm{k}_{2} / K_{\mathrm{m}}\right)$ of pro-UK reported by Collen et al. who suggested that pro-UK was a fully active enzyme (13). Recently the kinetics of plasminogen activation by two other mutants (Gly ${ }^{158}$ and $\mathrm{Glu}^{158}$ ) were studied by Nelles et al. and shown to follow Michaelis-Menten kinetics, but with a low catalytic efficiency (34). When calculated from their data this was $\sim 0.5 \%$ that of TC-UK. This low activity, relative to that previously reported by their laboratory for wild type n-pro-UK (13), was attributed to the absence of a positively charged Lys residue at position 158 in the mutants. The authors concluded that TC-UK generation was "not a prerequisite for the activation of plasminogen by pro-UK" at physiological concentrations of the substrate.

Alternatively, the high catalytic activities reported for proUK (13) could have been related to TC-UK generated in the reaction mixture. An excess of synthetic substrate (S2251) was used to inactivate plasmin in these experiments. This may not have been fully inhibitory, especially since the synthetic substrate product contains a highly reactive $\mathrm{COOH}$-terminal lysine. A much lower relative activity for pro-UK (6\%) was obtained by Ellis et al. who used dansylated aprotinin to inhibit plasmin and measured plasminogen activation by increases in fluorescence polarization (14). When the reactivity of highly purified, DFP-treated n-pro-UK was measured against plasminogen by SDS-PAGE in the presence of inhibitors to UK and plasmin a relative activity of $0.4 \%$ compared with TC-UK was found (15). This activity is comparable to that of the Lys ${ }^{158}$ mutants from the present study and from that reported by Nelles et al. (34), suggesting that the mutants reflect the true intrinsic activity of n-pro-UK. From this we concluded that physiological and even pharmacological plasmin generation by pro-UK is dependent on its activation to TC-UK.

The clot lysis experiments support the concept that TC-UK generation is important to fibrinolysis by n-pro-UK. Fibrinolysis in a plasma milieu by the mutants was only $0.5-1 \%$ that of n-pro-UK. In a previous study it was shown that clot lysis under these same conditions was accompanied by only $\sim 10 \%$ conversion of pro-UK to TC-UK, suggesting that lysis could be due to pro-UK itself (16). The present results indicate that pro-UK activation is largely responsible for determining the rate of clot lysis. At the same time it appears from the previous findings that only a small fraction of the total available reservoir of pro-UK seems to be used while the bulk remains intact.

The present findings contribute supportive data to a previously proposed mechanism that helps explain the fibrin dependence of plasminogen activation by pro-UK. This mechanism was based on the observation that clot lysis in plasma by pro-UK, in contrast to t-PA, was significantly potentiated by plasmin pretreatment of fibrin. Since this effect was inhibited by carboxypeptidase $B$, the plasmin-mediated potentiation was ascribed to the exposure of neo- $\mathrm{COOH}$-terminal lysines 
on fibrin. When plasminogen binds to these sites it is believed to undergo a particular conformational change that favors its selective activation by pro-UK (35). The present study shows that this property of pro-UK is shared by the mutants. Clot lysis by the mutants was similarly potentiated by plasmin pretreatment of fibrin or by the addition of UK or t-PA, both of which also induce the exposure of $\mathrm{COOH}$-terminal lysine residues on the $\alpha, \beta$, and $\gamma$ chains of fibrin(ogen) (40). In the case of plasmin, potentiation was essentially limited to an attenuation of the lag phase. This may be explained by the more limited effect of plasmin in exposing a finite number of $\mathrm{COOH}$-terminal lysine residues on fibrin that are eventually also exposed by the intrinsic activity of the mutants. Therefore, after a certain interval the lysis rates of the treated and untreated clots become parallel since they reflect the activity of the mutant against plasminogen bound to these sites. In the case of UK, and especially t-PA, there is a more sustained plasmin effect generating lysine termini at a more continuous rate, which results in a more sustained difference in lysis rates.

From the present findings it appears that the preferential activation of plasminogen bound to $\mathrm{COOH}$-terminal lysines on fibrin is a function of pro-UK itself. The absence of plasminogen or fibrinogen degradation in the plasma at the end of clot lysis attests to the relative resistance of the unbound plasminogen to activation by the mutants.

The mutants appeared to be synergistic in their fibrinolytic effect with t-PA as previously shown for n-pro-UK in vitro (37). The synergy of combinations of t-PA and n-pro-UK in fibrinolysis has been ascribed to their separate and complementary mechanisms of fibrin-dependent plasminogen activation (35). That is, in contrast to pro-UK, $t$-PA-induced lysis was found to be unresponsive to plasmin and carboxypeptidase B treatment of fibrin. This finding suggested that fibrinbound t-PA selectively activates plasminogen on undegraded fibrin. This plasminogen is bound to an internal lysine residue since $\mathrm{COOH}$-terminal lysines on fibrin are made available only after limited degradation by plasmin (35). The present finding that fibrinolysis by the mutants was also synergistic with t-PA indicates that this synergy is a function of pro-UK itself and is not fundamentally related to a potentiation of TC-UK generation by t-PA as was recently suggested (41).

In conclusion, this study supports the hypothesis that a selective interaction by pro-UK itself with plasminogen bound to certain $\mathrm{COOH}$-terminal lysines is involved in fibrinolysis by pro-UK. The findings also indicate that activation to TC-UK is largely responsible for the lysis. Little information on the mechanism by which TC-UK generation is brought about has been obtained. Under the experimental conditions it is likely that plasmin stabilized by fibrin against inhibition by $\alpha_{2}$-antiplasmin (42) is responsible. However, since pro-UK and t-PA appear to activate different fibrin-bound plasminogens (35), it remains to be shown whether an internal or $\mathrm{COOH}$-terminal lysine-bound plasmin is responsible for the activation of proUK. The higher reactivity of the mutant against $\mathrm{COOH}$-terminal lysine-bound plasminogen, as evidenced by the potentiating effect of plasmin pretreatment and of UK and t-PA, makes it possible that the intrinsic activity of pro-UK may initiate a cycle resulting in the mutual activation of plasminogen and pro-UK. However, a relatively high concentration of pro-UK would still seem to be required to initiate this positive feedback cyclical reaction. Therefore, the internal lysine-bound plasmin generated by t-PA may be more readily available for the acti- vation of pro-UK bound to its substrate at a $\mathrm{COOH}$-terminal lysine residue.

\section{References}

1. Williams, J. R. B. 1951. The fibrinolytic activity of urine. Br. J. Exp. Pathol. 32:530-539.

2. Husain, S. S., B. Lipinski, and V. Gurewich. 1979. Isolation of plasminogen activators useful as therapeutic and diagnostic agents (single-chain, high fibrin affinity urokinase). U.S. Patent No. 4381346 (filed November 13, 1979; issued April 26, 1983).

3. Husain, S. S., V. Gurewich, and B. Lipinski. 1981. Purification of a new high molecular weight form of urokinase from urine. Thromb. Haemostasis. 46:11.

4. Husain, S. S., V. Gurewich, and B. Lipinski. 1983. Purification and partial characterization of a single-chain, high molecular weight form of urokinase from human urine. Arch. Biochem. Biophys. 220:31-38.

5. Wun, T. C., L. Ossowski, and E. Reich. 1982. A proenzyme form of urokinase. J. Biol. Chem. 257:7262-7268.

6. Nielsen, L. S., J. G. Hansen, L. Skriver, E. L. Wilson, K. Kaltoft, J. Zeuthen, and K. Dano. 1982. Purification of zymogen to plasminogen activator from human glioblastoma cells by affinity chromatography with monoclonal antibody. Biochemistry. 21:6410-6414.

7. Wun, T. C., W. D. Schleuning, and E. Reich. 1982. Isolation and characterization of urokinase from human plasma. J. Biol. Chem. 257:3276-3283.

8. Eaton, D. L., R. W. Scott, and J. B. Baker. 1984. Purification of human fibroblast urokinase proenzyme and analysis of its regulation by proteases and protease nexin. J. Biol. Chem. 259:6241-6247.

9. Gunzler, W. A., G. J. Steffens, F. Otting, G. Buse, and L. Flohe. 1982. Structural relationship between human high and low molecular mass urokinases. Hoppe-Seyler's Z. Physiol. Chem. 363:133-141.

10. Gurewich, V., R. Pannell, S. Louie, P. Kelley, R. L. Suddith, and R. Greenlee. 1984. Effective and fibrin-specific clot lysis by a zymogen precursor form of urokinase (pro-urokinase). A study in vitro and in two animal species. J. Clin. Invest. 73:1731-1739.

11. Pannell, R., and V. Gurewich. 1985. Pro-urokinase (PUK): characterization of its enzymatic activation. Thromb. Haemostasis. 54:102.

12. Ichinose, A., K. Fujikawa, and S. Tadakazu. 1986. The activation of pro-urokinase by plasma kallikrein and its inactivation by thrombin. J. Biol. Chem. 261:3486-3489.

13. Collen, D., C. Zamarron, H. R. Lijnen, and M. Hoylaerts. 1986. Activation of plasminogen by pro-urokinase. II. Kinetics. $J$. Biol. Chem. 261:1259-1266.

14. Ellis, V., M. F. Skully, and V. V. Kakkar. 1987. Plasminogen activation by single-chain urokinase in functional isolation. J. Biol. Chem. 262:14998-15003.

15. Pannell, R., and V. Gurewich. 1987. The activation of plasminogen by single-chain urokinase or by two-chain urokinase. A demonstration that single chain urokinase has a low catalytic activity (prourokinase). Blood. 69:22-26.

16. Pannell, R., and V. Gurewich. 1986. Pro-urokinase. A study of its stability in plasma and a mechanism for its selective fibrinolytic effect. Blood. 67:1215-1223.

17. Kohno, T., P. Hopper, J. S. Lillquist, R. L. Suddith, R. Greenlee, and D. T. Moir. 1984. Kidney plasminogen activator. A precursor form of human urokinase with high fibrin affinity. Biotechnology. 2:628-634.

18. Favaloro, J., R. Treisman, and R. Kamen. 1980. Transcription maps of polyoma virus-specific RNA: analysis by two-dimensional nuclease S1 gel mapping. Methods Enzymol. 65:718-749.

19. Aviv, H., and P. Leder. 1972. Purification of biologically active globin messenger RNA by chromotography on oligothymidylic acidcellulose. Proc. Natl. Acad. Sci. USA. 69:1408-1412. 
20. Moir, D. T., J. Mao, J. W. Schumm, G. F. Vovis, B. L. Alford, and A. Taunton-Rigby. 1982. Molecular cloning and characterization of double-stranded cDNA coding for bovine chymosin. Gene (Amst.). 19:127-138.

21. Benton, W. D., and R. W. Davis. 1977. Screening gt recombinant clones by hybridization to single plaques in situ. Science (Wash. DC). 196:180-182.

22. Holmes, W. E., C. Pennica, M. Blaber, M. W. Rey, W. A. Guenzler, G. J. Steffens, and H. L. Heyneker. 1985. Cloning and expression of the gene for pro-urokinase in Escherichia coli. Biotechnology. 3:923-929.

23. Sanger, F., S. Nicklen, and A. R. Coulson. 1977. DNA sequencing with chain-terminating inhibitors. Proc. Natl. Acad. Sci. USA. 74:5463-5467.

24. Maxam, A. M., and W. Gilbert. 1980. Sequencing end-labeled DNA with base-specific chemical cleavages. Methods Enzymol. 65:499-560.

25. Mulligan, R. C., and P. Berg. 1981. Selection for animal cells that express the E. coli gene coding for xanthine-guanine phosphoribosyltransferase. Proc. Natl. Acad. Sci. USA. 78:2072-2076.

26. Urlaub, G., E. Kas, A. M. Carothers, and L. A. Chasin. 1983. Deletion of the diploid dihydrofolate reductase locus from cultured mammalian cells. Cell. 33:405-412.

27. Subramani, S., R. Mulligan, and P. Berg. 1981. Expression of the mouse dihydrofolate reductase complementary deoxyribonucleic acid in Simian Virus 40 vectors. Mol. Cell. Biol. 1:854-864.

28. Crouse, G. F., R. N. MeEwan, and M. L. Pearson. 1983. Expression and amplification of engineered mouse dihydrofolate reductase minigenes. Mol. Cell. Biol. 3:257-266.

29. Graham, F. L., and A. J. van der Eb. 1973. A new technique for the assay of infectivity of human adenovirus 5 DMA. Virology. 52:456-467.

30. Kaufman, R., and P. Sharp. 1982. Amplification and expression of sequences cotransfected with a modular dihydrofolate reductase cDNA gene. J. Mol. Biol. 159:601-621.

31. Lowry, O. H., N. J. Rosenbrough, A. L. Farr, and R. J. Randt. 1951. Protein measurements with the Folin phenol reagent. J. Biol. Chem. 193:265-275.
32. Castellino, J., and J. R. Powell. 1981. Human plasminogen. Methods Enzymol. 80:365-378.

33. Gurewich, V., and R. Pannell. 1987. Inactivation of single chain urokinase (pro-urokinase) by thrombin and thrombin-like enzymes. Relevance of the finding to the interpretation of fibrin binding experiments. Blood. 69:769-772.

34. Nelles, L., R. H. Lijnen, D. Collen, and W. Holmes. 1987. Characterization of recombinant single chain urokinase-type plasminogen activator mutants produced by site-specific mutagenesis of Lysine 158. J. Biol. Chem. 262:5682-5689.

35. Pannell, R., J. Black, and V. Gurewich. 1988. The complementary modes of action of tissue plasminogen activator (t-PA) and prourokinase (pro-UK) by which their synergistic effect on clot lysis may be explained. J. Clin. Invest. 81:853-859.

36. Gurewich, V. 1987. Experiences with pro-urokinase and potentiation of its fibrinolytic effect by urokinase and by tissue plasminogen activator. J. Am. Coll. Cardiol. 10(5):16B-21B.

37. Gurewich, V., and R. Pannell. 1986. A comparative study of the efficacy and specificity of tissue plasminogen activator and prourokinase: demonstration of synergism and of different thresholds of non-selectivity. Thromb. Res. 44:217-228.

38. Collen, D., F. DeCock, E. Demarsin, H. R. Lijnen, and D. C. Stump. 1986. Absence of synergism between tissue-type plasminogen activator (t-PA), single-chain urokinase-type plasminogen activator (scu-PA) and urokinase on clot lysis in a plasma milieu in vitro. Thromb. Haemostasis. 56(1):35-39.

39. Berenbaum, M. C. 1977. Synergy, additivism and antagonism in immunosuppression: a critical review. Clin. Exp. Immunol. 28:1-18.

40. Harpel, P. C., T.-S. Chang, and E. Verderber. 1985. Tissue plasminogen activator and urokinase mediate the binding of Glu-plasminogen to plasma Fibrin I: evidence for new binding sites in plasmin-degraded Fibrin I. J. Biol. Chem. 260:4432-4440.

41. Fry, E. T. A., D. L. Mack, and B. E. Sobel. 1988. Synergistic thrombolysis in vitro with t-PA and scu-PA. Clin. Res. 36:363a. (Abstr.)

42. Lijnen, H. R., and D. Collen. 1982. Interaction of plasminogen activators and inhibitors with plasminogen and fibrin. Semin. Thromb. Hemostasis. 8:2-9. 